\title{
Research of Book Positioning System Based on Wireless Sensor Network
}

\author{
Huan Wang ${ }^{1}$ and Kai Wang ${ }^{2}$ \\ ${ }^{1}$ Library of Harbin University of Science and Technology, Harbin, China \\ ${ }^{2}$ Department of Exercise Science and Health, Harbin Institute of Physical \\ Education, Harbin, China \\ Wanghuan220292@sina.com
}

\begin{abstract}
In order to meet the need of book management in smart library and achieve the positioning of perception node for book position, ZigBee wireless smart network technology is adopted to achieve the information interaction of book information and book management, and RFID technology is used to provide communication perception platform for monitoring book node. Least square method and RSSI (Received signal strength indication) are used to achieve the optimized $3 d$ positioning for book node. The simulation result shows that the amount of calculation for the algorithm is small, whose positioning accuracy reaches $1.21 \%$.
\end{abstract}

Keywords: RFID; Zigbee; sensor Network; Book Management

\section{Introduction}

With the rapid development of RFID and ZigBEE wireless sensor network group technology and calculation of route, low-consumption and low-cost multiple-function sensor node and route technology are applied in many fields [1-4], including smart group network, smart industry, military investigation, mine check, and medical supervision and so on. Due to the proposal of industrial 4.0 technologies, large data cloud computing and loT technology based on wireless sensor network technology become the essential trend for the development of smart network in the future. In smart network, rapidly and accurately confirming the position of sensor node in the network and acquiring the senor data are the core problems of group network check technology [5-6].

The thesis designs book management system platform based on RFID and ZigBee wireless positioning system; transforms the positioning problem of sensor node to optimization problem; adopts least square method to solve the optimization to improve the efficiency of library management system and reduce the time-delay for book search and management, laying theoretical foundation and practical experience for the follow-up smart library.

\section{General Plan Design}

Library management platform is the comprehensive system which collects the functions of information sensor, dynamic decision-making and planning, and behavior execution. In the process of library management, the monitoring site in the complicated conditions---book node is often browsed and moved by readers, so the node information of monitoring site is changeable continuously. Smart optimizing calculation $[7,8]$ should be used to acquire the perception information of monitoring site.

The book positioning and perception system designed in this thesis mainly includes ZigBee wireless module, RFID sensor module, extension interface, and electrical source management module. Its structure is as shown in Figure 1. 


\begin{tabular}{|c|c|}
\hline $\begin{array}{c}\text { RSSI } \\
\text { positioning } \\
\text { algorithm }\end{array}$ & $\begin{array}{c}\text { Ethernet } \\
\text { communication } \\
\text { module }\end{array}$ \\
\hline \multicolumn{2}{|c|}{ book perception node } \\
MSP430 \\
\hline RFID reader & $\begin{array}{c}\text { ZigBee wireless } \\
\text { module }\end{array}$ \\
\hline
\end{tabular}

\section{Figure 1. Structure of Book Positioning and Perception System}

Considering the low-power dissipation requirement of system platform, we select MSP430F2618 SCM and CC2430 of node radio frequency chip from TI company, which support 2.4GHz EEE802.15.4/ZigBee protocal. It uses wireless sensor network to percept its own space position, and it uses the label produced by Philips to store book information and to achieve the assistant positioning and searching operation of wireless network.

\section{Hardware Design}

The system includes three parts. In order to meet the requirements of low power dissipation and environment of library, wiring for a large scale cannot be used. Various nodes should be distributed. Major function modules include 1) RFID module including reader and signal processing circuit, 2) ZigBee module including the data collection for sensor output module and communication with MSP430 SEM, and 3) ZigBee node group network module.

In order to achieve the accurate positioning of book perception node, the method of combination of ZigBee terminal and RFID reader can be adopted. It uses the principal and subordinate method of combination of terminal node and route node to shape the wireless network. The network structure is as shown in Figure 2.

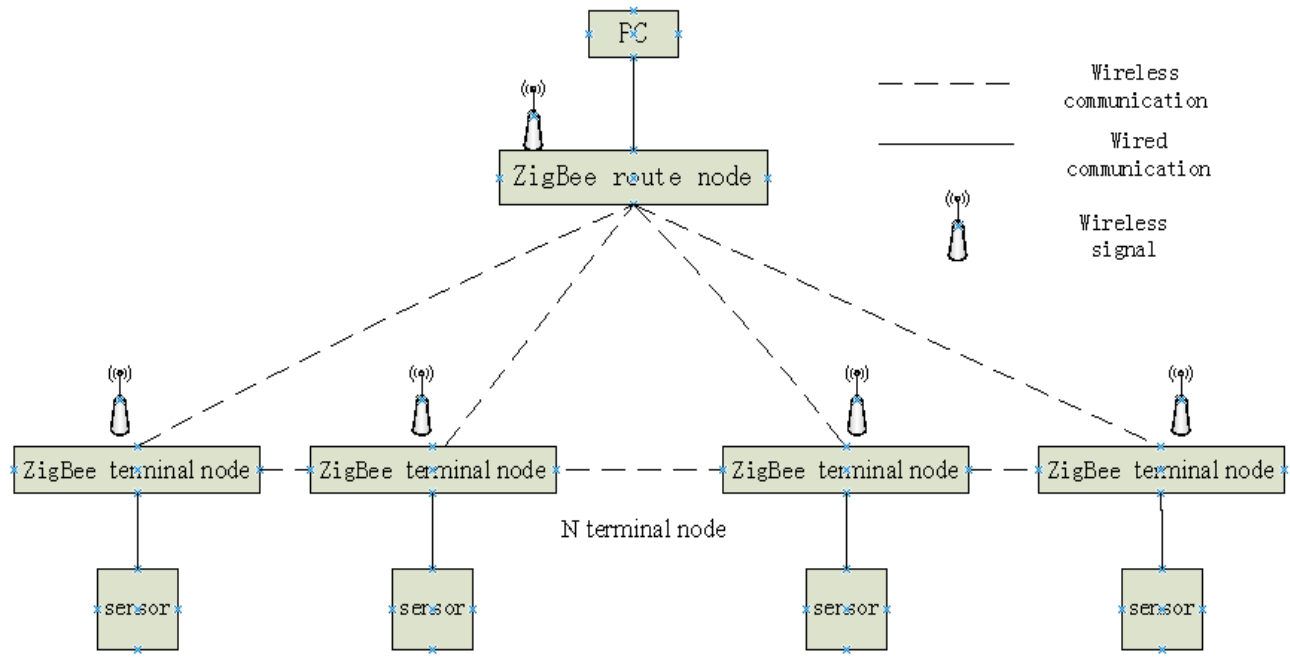

Figure 2. Structure of Wireless Network

CC2530 is a true system solution plan on the chip. CC2530 has been improved a lot than CC2430 in internal memory, size, and EF function. The EF function of this chip is better, whose flash memory volume doubles with smaller package size and more support protocols. The outdoor longest transmission distance of the designed sensor node on the 
basis of this chip reaches $400 \mathrm{M}$, which completely complies with the test requirement within the greenhouse. CC2530 can transform running mode according to different needs, letting it adapt to the low power dissipation system such as test of temperature and humidity of greenhouse. As the integrated circuit in the CC2530 chip is comprehensive, periphery circuit is relatively simple. CC2530 periphery circuit is as shown in Figure 3.

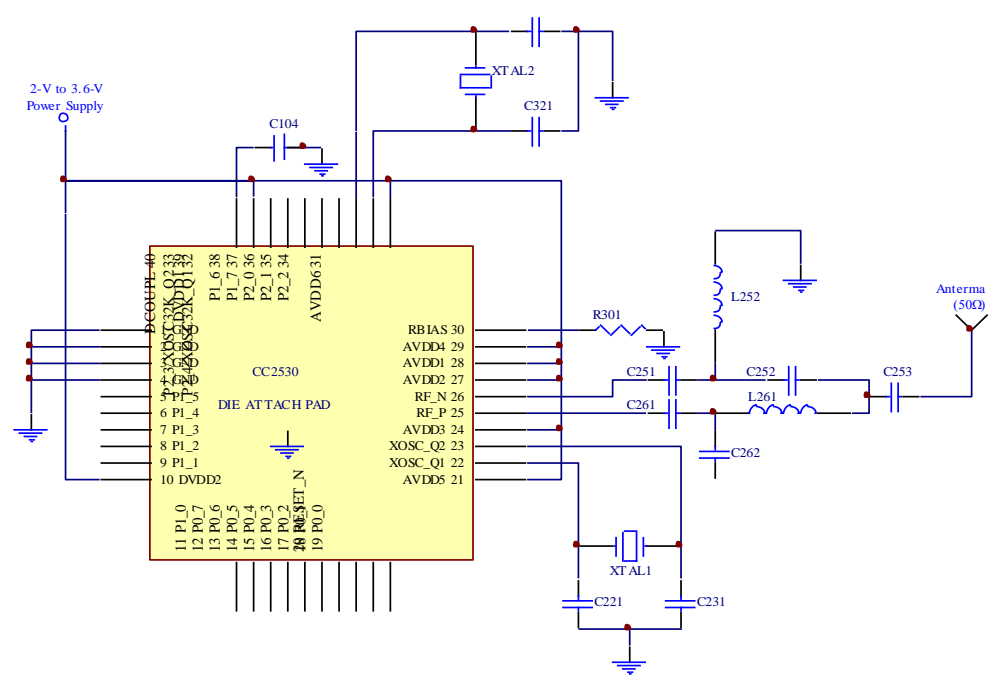

Figure 3. CC2530 Periphery Circuit Diagram

\section{Design of Book Positioning and Perception Node Software}

Design of book positioning and perception node software includes data collection and signal processing procedure, serial interface communication procedure, and ZigBee protocol stack procedure. Module design includes initial module, data collection module, and digital filtration module and so on. The core part is the achievement of ZigBee network node software design and positioning calculation.

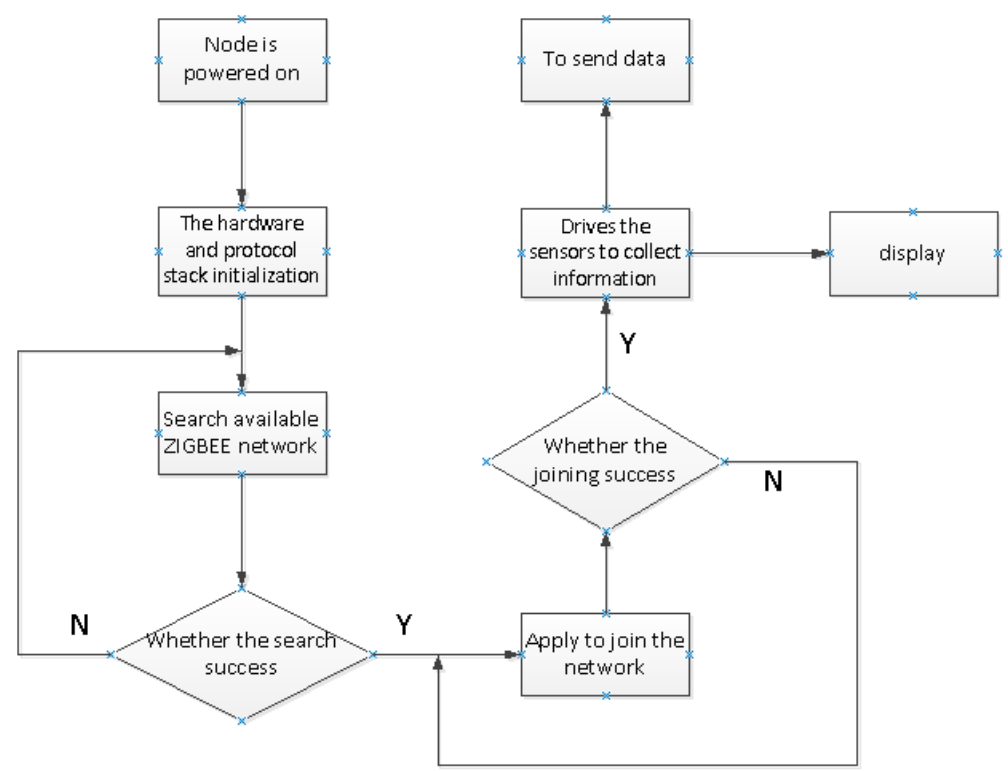

Figure 4. Flow Chart of Terminal Node Software 
After the coordinator node energizes, first initialize the hardware and protocol stack, search information channel and assess free information channel, and select information channel and establish ZigBee network. When a node applies to join in the network, it is allowed to join and it will be distributed a 16-site short network site. Wait the order for collecting the data. Then send the received all of data to PC through serial interface communication to prepare for data analysis and data storage. The flow chart of coordinator node software is shown in Figure 5.

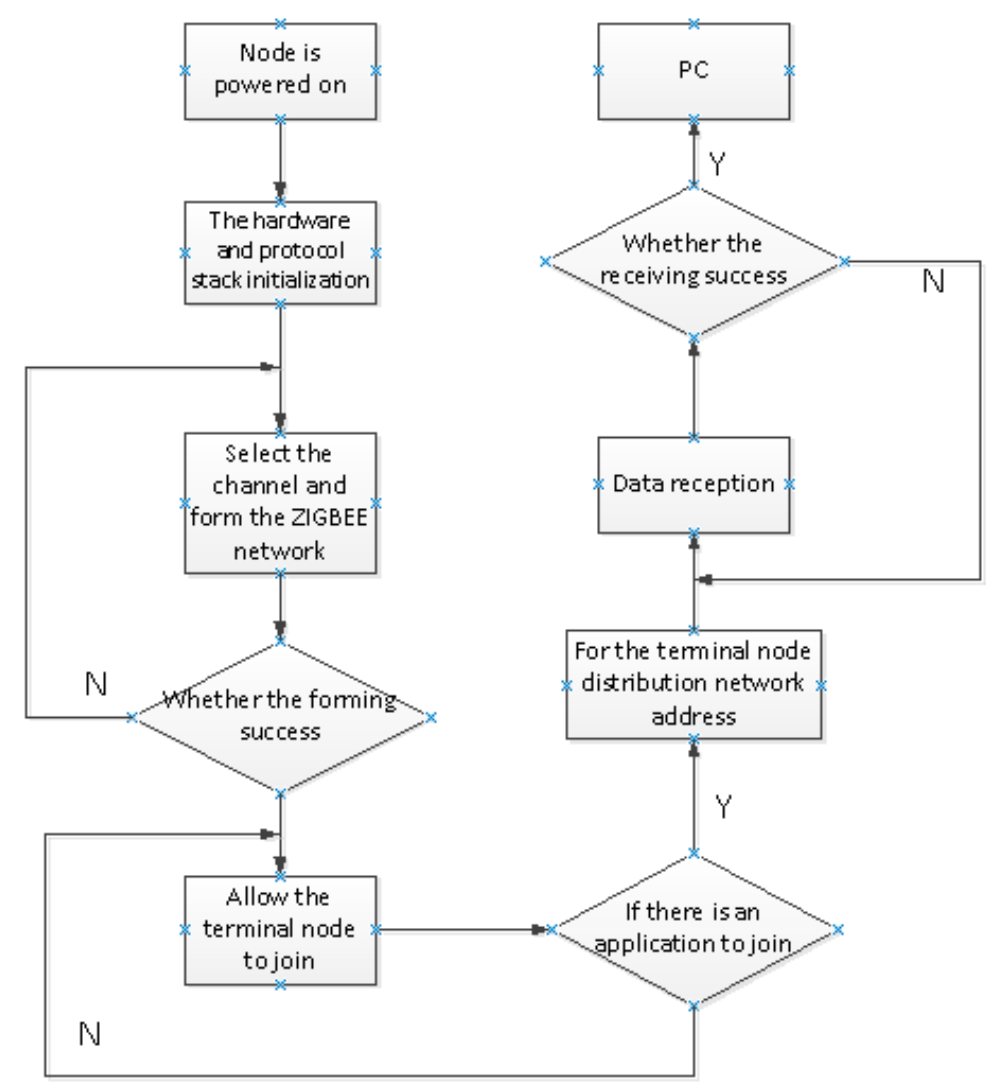

Figure 5. Flow Chart of Coordinator Node Software

Initiation includes electric initiation on the sensor and wireless chip distributed ID. ZigBee is connected with PC with the help of Ethernet, which is monitored and controlled by PC. The processor CC2430 of perception and positioning system is connected with upper monitor software with the help of expanded Ethernet, which sends the collected positioning data to user interface. Therefore, books in the library can be positioned and managed conveniently.

\section{Calculation of Book Positioning}

The distance measurement technology based on RSSI uses the principle that wireless signal reduces regularly with the increasing of the distance to measure the distance between nodes. The relationship between RSSI and $d$ can be signed as:

$$
R S S I=-(10 n \lg d+A)
$$

In the formula, $n$ represents signal transmission constant, which is also called transmission constant; $d$ is the distance between signal and sender; $A$ represents the signal strength when it keeps a distance of 1 meter with the sender. The accuracy of 
distance measurement is obviously affected by the practical value of $n$ and $A \cdot n$ describes the parameter that signal strength reduces gradually with the increasing of distance, and the value of $n$ rests on concrete environment. In order to get the optimized value of $n$, all of the nodes for reference could be put first, and then try to use different values of $n_{-}$Index to find the best value of $n$ for this concrete environment.

The calculation of the thesis is the solution to the problem of book perception node positioning, which processes the information of received signal strength according to Gaussian distribution data processing principle; selects suitable routine to position; designs the target function for positioning; uses the stationary node on the bookshelf to percept and position; transforms the positioning problem into optimization problem; and uses optimization calculation to provide the position of book perception node at the least value of error of system positioning.

The coordinate of the stationary node is got by the positioning of CC2430 and RFID stationary point reader. If there were $N$ nodes in the sensor network with $n$ unknown nodes and $m$ known-position nodes, the strength volume of undetermined label for $n$ AP nodes are:

$$
P=\left[\begin{array}{llll}
A P_{1} & A P_{2} & \ldots & A P_{n}
\end{array}\right]
$$

Its corresponding coordinate is

$$
X=\left[\begin{array}{llll}
X_{1} & X_{2} & \cdots & X_{n}
\end{array}\right]
$$

$X_{i}=\left[\begin{array}{llll}X_{i 1} & X_{i 2} & \ldots & X_{i k}\end{array}\right]$ Represents k dimensional space. In this thesis, $k=3$.

According to the coordinate of stationary nodes, RSSS is used to measure the coordinate of book perception node

$$
\hat{X}=\left[\begin{array}{llll}
\hat{X}_{1} & \hat{X}_{2} & \ldots & \hat{X}_{n}
\end{array}\right]
$$

According to formula (4), the Euclidean distance of the undetermined node and datum node are

$$
E_{t}=\sqrt{\sum_{i=1}^{n}\left(X_{i}-\hat{X}_{i t}\right)^{2}}, t=1,2,3
$$

It is known from formula (1) that if RSSI value between datum node and blind node is known, the distance between two nodes can be estimated. However, there might be different signal disturbance under different environments, so adopting RSSI value between nodes must have deviation. At the moment, the measured distance can be corrected by using traditional least square method in accordance with the special environment [8]. In this way, the corrected distance can be got, and then the coordinate of the blind node can be estimated accurately. The concrete steps are as follows:

(1) According to the situation that books are put, adopt the method of rectangle positioning to conserve the coordinates of stationary node $\left(x_{i}, y_{i}, z_{i}\right)$

(2) According to formula (5), the distance between undetermined book perception node and stationary node can be counted;

(3) The relationship between practical distance $X_{i}$ and estimated distance $\hat{X}_{i}$ can be got with the help of least square method. The fitted relationship is $\hat{X}_{i}=a X_{i}+b$;

(4) Least square estimated value is got

$$
J=\sum_{k=1}^{\infty}(e(k))^{2}=\sum_{k=1}^{\infty}\left(a X_{k}+b-\hat{X}_{k}\right)^{2}
$$


If the value is the smallest, the fitted value of $a$ and $b$ are got;

(5) With the help of fitted relationship, the coordinates of $\hat{X}$ of the undetermined book perception node can be counted.

\section{Simulation Research}

The calculations for wireless sensor network node positioning mainly include the calculation based on distance measurement and non-distance measurement calculation. The common calculations of distance measurement include RSSI, TOA, TDOA, and AOA and so on [9]. The thesis adopts RSSI and least square method to conduct positioning experiment to book node.

The experiment distributes stationary nodes in the experiment space in accordance with rectangle form. Each stationary node includes $3 \mathrm{~d}$ coordinates. In the experiment, a series of bookshelves are selected to be positioning experiment nodes. The $3 \mathrm{~d}$ coordinates of the stationary nodes are set as $(10,2.5,2),(10,2.5,0.5),(10,4,2),(10,4,0.5)$.

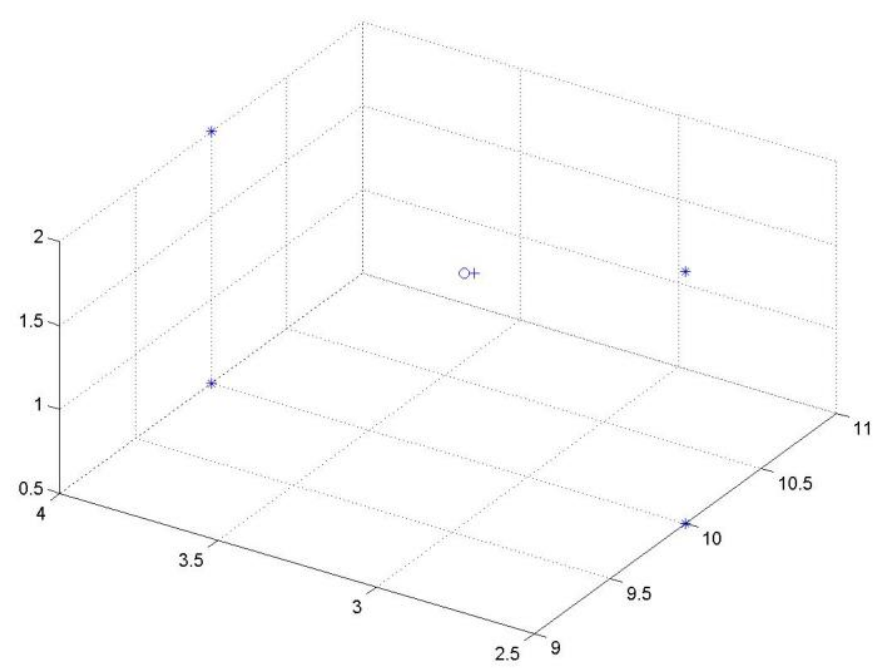

Figure 6. Result of Positioning Simulation

In Figure 6, "+" is the optimized book perception coordinate; "o" is the real coordinate; "*" is the coordinate of RFID readers of the stationary nodes. The deviation formula is used to assess the deviation between the optimized coordinate and real coordinate. The deviation is $1.21 \%$, which completely meets the deviation need of book monitoring.

\section{Conclusions}

The thesis uses ZigBee wireless sensor network and module group network thought to design and develop the book positioning and perception system, whose maintenance is strong, shortening the period for development. Meanwhile, the thesis uses least square method to optimize and correct RSSI positioning calculation, reducing the signal interference in the monitoring of nodes. Simulation and experiment results show that various sensor nodes can transmit data rapidly and accurately with high reliability, which achieves the intelligence of book management and raise the efficiency of book search. 


\section{Acknowledgments}

This paper is partially supported by Colleges and Universities in Heilongiiang Province Commission Project (2013-B-045).

\section{References}

[1] D. Moeinfar, H. Shamsi and F. Nafar, "Design and Implementation of a Low-Power Active RFID for Container Tracking at $2.4 \mathrm{GHz}$ Frequency",Journal of Advances in Internet of Things, vol. 2, (2012), pp. 13-22.

[2] M. Ma and G. Feng, "Based on Zigbee Technology Greenhouse Monitoring System”, Journal of Advances in Intelligent Systems and Computing, vol. 191, (2013), pp. 737-740.

[3] I. Akyildiz, W. Su and Y Sankarasubramaniam, "A Survey on Sensor Networks", IEEE Communications Magazine, vol. 40, no. 8, (2002), pp. 102- 114

[4] Z. Li and C. Duan, "Zigbee2007/PRO Protocol Stack Test and Practice", Beijing: Beihang University Press, (2009).

[5] Q. Zhang, Q. Di, G. Xu and X. Qiu, “A RSSI Based Localization Algorithm for Multiple Mobile Robots", 2010 International Conference on Computer, Mechatronics, Control and Electronic Engineering (CMCE 2010).

[6] H. Caijun, "The Applied Research Of Zigbee Wireless Sensor Network Technology In The Industrial Control System", Hunan University, 2010Guizeng Wang, Hao Ye, Principal component analysis and partial least square method[M], Beijing: Tsinghua University press, (2012), pp. 7-9.

[7] C. Xu, F. Kangling and L. Xiaohui, "The Design of Data Collection System Based on Zigbee", Journal of Hunan University of Technology, vol. 22, no. 6, (2008), pp. 59-61.

[8] J. Zhang and L. Zhang, "Research on distance measurement based on RSSI of ZigBee", Chinese Journal of Sensors and Actuators, vol. 22, no. 2, (2009), pp. 285-286.

[9] G. Qingmin, H. Huan and S. Ruijie, "Application Of Wireless Sensor Network Based On Zigbee To Substation Monitoring System”, North China Water Conservancy and Hydropower's Journal, vol. 2, no. 1, (2010), pp. 53-56. 
International Journal of Future Generation Communication and Networking Vol. 8, No. 5 (2015) 